\title{
Global exponential stability of nonautonomous neural network models with continuous distributed delays
}

\author{
Salete Esteves ${ }^{\ddagger}$, Elçin Gökmen ${ }^{\dagger}$ and José J. Oliveira* \\ (†) Departamento de Informática e Matemática, EsACT - IPB, \\ 5370-326 Mirandela, Portugal \\ e-mail: saleteesteves@ipb.pt \\ (†) Department of Mathematics, Faculty of Science, Muğla Sitkı Koçman University, \\ 4800 Muğla, Turkey \\ e-mail: elcingokmen@hotmail.com \\ (*) Departamento de Matemática e Aplicações and CMAT, Escola de Ciências, \\ Universidade do Minho, Campus de Gualtar, 4710-057 Braga, Portugal \\ e-mail: jjoliveira@math.uminho.pt
}

\begin{abstract}
For a family of non-autonomous differential equations with distributed delays, we give sufficient conditions for the global exponential stability of an equilibrium point. This family includes most of the delayed models of neural networks of Hopfield type, with time-varying coefficients and distributed delays. For these models, we establish sufficient conditions for their global exponential stability. The existence and global exponential stability of a periodic solution is also addressed. A comparison of results shows that these results are general, news, and add something new to some earlier publications.
\end{abstract}

Keywords: Hopfield neural network, BAM neural network, Time-varying coefficient, Distributed time delay, Periodic solution, Global exponential stability.

2012 Mathematics Subject Classification: 34K13, 34K20, 34K25, 92B20.

\section{Introduction}

In the last decades, retarded functional differential equations (FDEs) have attracted the attention of an increasing number of scientists due to their potential applications in different sciences. Differential equations with delays have served as models in population dynamics, ecology, epidemiology, disease modeling, and neural networks.

Neural network models possess good potential applications in areas such as contentaddressable memory, pattern recognition, signal and image processing and optimization (see [2], [15], [18], [19], and references therein).

In 1983, Cohen and Grossberg [5] proposed and studied the artificial neural network described by a system of ordinary differential equations

$$
x_{i}^{\prime}(t)=-k_{i}\left(x_{i}(t)\right)\left[b_{i}\left(x_{i}(t)\right)-\sum_{j=1}^{n} a_{i j} f_{j}\left(x_{j}(t)\right)\right], \quad i=1, \ldots, n
$$

and, in 1984, Hopfield [9] studied the particular situation of (1.1) with $k_{i} \equiv 1$,

$$
x_{i}^{\prime}(t)=-b_{i} x_{i}(t)+\sum_{j=1}^{n} a_{i j} f_{j}\left(x_{j}(t)\right), \quad i=1, \ldots, n .
$$


In 1988, Kosko presented a kind of neural networks, which is called bidirectional associative memory (BAM) neural network, [13],

$$
\left\{\begin{array}{l}
x_{i}^{\prime}(t)=-x_{i}(t)+\sum_{j=1}^{n} a_{i j} f\left(y_{j}(t)\right)+I_{i} \\
y_{i}^{\prime}(t)=-y_{i}(t)+\sum_{j=1}^{n} b_{j i} f\left(x_{i}(t)\right)+J_{i}
\end{array}\right.
$$

The finite switching speed of the amplifiers, communication time, and process of moving images led to the use of time-delays in models (1.1), (1.2), and (1.3), arising the delayed neural network models. In the applications of delayed neural networks to some practical problems, stability plays an important role. It is well known that delays can affect the dynamic behavior of neural networks (see [1], [14]). For this reason, stability of delayed neural networks has been investigated extensively. There are many important results on static (equilibrium-type) attractors of neural networks (see [2], [3], [7], [10], [15], [16], and the references therein), but it is well known that non-static attractors, such as periodic oscillatory behavior, are also an important aspect (see [4], [11], [17], [20], [21], and the references therein).

In the literature, the usual approach to analyze the stability property is to construct a suitable Lyapunov functional for a concrete n-dimensional FDE and then to derive sufficient conditions ensuring stability. However, constructing a Lyapunov functional is not an easy task and, frequently, a new functional is required for each model under consideration. In quite an unusual way, our techniques (see [6], [7], [15], [16]) do not involve Lyapunov functionals, and our method applies to general systems.

This paper is organized as follows: In Section 2, we briefly present the phase space for FDEs written in abstract form as $x^{\prime}(t)=f\left(t, x_{t}\right)$, then we define the global exponential stability of a FDE, and finally we establish a general condition for the boundedness of solutions of $x^{\prime}(t)=f\left(t, x_{t}\right)$. In Section 3, we present the results on global exponential stability of a general class of nonautonomous delay differential equations, which includes most of neural network models. In Section 4, we prove the existence and global exponential stability of a periodic solution of a periodic general Hopfield neural network type model. Finally, in Section 5, we illustrate the results with well-known nonautonomous n-dimensional neural network models and we compare our results with the literature, showing the advantage of our method when applied to several different models, such as Hopfield or BAM neural network models.

\section{Preliminaries}

For $a, b \in \mathbb{R}$ with $b>a$ and $n \in \mathbb{N}$, we denote by $C\left([a, b] ; \mathbb{R}^{n}\right)$ the vector space of continuous functions $\varphi:[a, b] \rightarrow \mathbb{R}^{n}$, equipped with the supremum norm $\|\cdot\|$ relative to the $\max$ norm $|\cdot|$ in $\mathbb{R}^{n}$, i.e., $\|\varphi\|=\sup _{a \leq \theta \leq b}|\varphi(\theta)|$ for $\varphi \in C\left([a, b] ; \mathbb{R}^{n}\right)$, where $|x|=\max _{i=1, \ldots, n}\left|x_{i}\right|$ for $x=\left(x_{1}, \ldots, x_{n}\right) \in \mathbb{R}^{n}$. For $c \in \mathbb{R}$, we use $c$ to denote the constant function $\varphi(\theta)=c$ in $C\left([a, b] ; \mathbb{R}^{n}\right)$. A vector $d=\left(d_{1}, \ldots, d_{n}\right) \in \mathbb{R}^{n}$ is said to be positive if $d_{i}>0$ for $i=1, \ldots, n$, and in this case we write $d>0$. 
In the space $C_{n}:=C\left([-\tau, 0] ; \mathbb{R}^{n}\right)$, for $\tau>0$, consider FDEs,

$$
x^{\prime}(t)=f\left(t, x_{t}\right), \quad t \geq 0,
$$

where $f:[0,+\infty) \times C_{n} \rightarrow \mathbb{R}^{n}$ is a continuous function and, as usual, $x_{t}$ denotes the function in $C_{n}$ defined by $x_{t}(\theta)=x(t+\theta),-\tau \leq \theta \leq 0$. It is well-known that, assuming the Banach space $C_{n}$ as the phase space of (2.1), the standard existence, uniqueness, and continuous type results are valid (see [8]). We always assume that $f$ is regular enough in order to have uniqueness of solutions for the initial value problem. The solution of (2.1) with initial condition $x_{t_{0}}=\varphi$, for $t_{0} \geq 0$ and $\varphi \in C_{n}$, is denoted by $x\left(t, t_{0}, \varphi\right)$. For $\omega>0$ and $\varphi \in C_{n}$, we write $x_{\omega}(\varphi)$, or just $x_{\omega}$ if there is no confusion, to denote the function in $C_{n}$ defined by $x_{\omega}(\varphi)(\theta)=x(\omega+\theta, 0, \varphi), \theta \in[-\tau, 0]$.

Definition 2.1. The solution $x(t, 0, \bar{\varphi})$ of $(2.1)$, with $\bar{\varphi} \in C_{n}$, is said globally exponentially stable if there are $\varepsilon>0$ and $M \geq 1$ such that

$$
|x(t, 0, \varphi)-x(t, 0, \bar{\varphi})| \leq M e^{-\varepsilon t}\|\varphi-\bar{\varphi}\|, \quad \forall t \geq 0, \forall \varphi \in C_{n} .
$$

Definition 2.2. The system (2.1) is said globally exponentially stable if there are $\varepsilon>0$ and $M \geq 1$ such that

$$
\left|x\left(t, 0, \varphi_{1}\right)-x\left(t, 0, \varphi_{2}\right)\right| \leq M e^{-\varepsilon t}\left\|\varphi_{1}-\varphi_{2}\right\|, \quad \forall t \geq 0, \forall \varphi_{1}, \varphi_{2} \in C_{n} .
$$

In [6], a relevant result on the boundedness of solutions for the general FDE (2.1) was established. For convenience of the reader, we put the proof here.

Lemma 2.1. [6] Consider equation (2.1) with the continuous functions $f=\left(f_{1}, \ldots f_{n}\right)$ satisfying:

(H) for all $t \geq 0$ and $\varphi \in C_{n}$ such that $|\varphi(\theta)|<|\varphi(0)|$ for $\theta \in[-\tau, 0)$, then $\varphi_{i}(0) f_{i}(t, \varphi)<0$ for some $i \in\{1, \ldots, n\}$ such that $|\varphi(0)|=\left|\varphi_{i}(0)\right|$.

Then, all solutions of (2.1) are defined and bounded for $t \geq 0$. Moreover, if $x(t)=$ $x(t, 0, \varphi)$, with $\varphi \in C_{n}$, is a solution of $(2.1)$, then $|x(t)| \leq\|\varphi\|$ for all $t \geq 0$.

Proof. Let $x(t)$ be the solution of $(2.1)$ on $[-\tau, a)$, for some $a>0$, such that $x_{0}=\varphi$ with $\varphi \in C_{n}$.

Suppose that there exists $t_{1}>0$ such that $\left|x\left(t_{1}\right)\right|>\|\varphi\|$, and define

$$
T=\min \left\{t \in\left[0, t_{1}\right]:|x(t)|=\max _{s \in\left[0, t_{1}\right]}|x(s)|\right\} .
$$

We have $|x(T)|>\|\varphi\|$ and

$$
|x(t)|<|x(T)|, \quad \text { for } t \in[0, T) .
$$

Hence, $\left|x_{T}(\theta)\right|=|x(T+\theta)|<|x(T)|$ for $\theta \in[-\tau, 0)$. By $(\mathbf{H})$, there is $i \in\{1, \ldots, n\}$ such that $|x(T)|=\left|x_{i}(T)\right|$ and $x_{i}(T) f_{i}\left(T, x_{T}\right)<0$. Suppose that $x_{i}(T)>0$ (the situation $x_{i}(T)<0$ is analogous). Since $x_{i}(t) \leq|x(t)|<x_{i}(T)$ for $t \in[-\tau, T)$, then $x_{i}^{\prime}(T) \geq 0$. On the other hand, from (2.1) we have $x_{i}^{\prime}(T)=f_{i}\left(T, x_{T}\right)<0$, which is a contradiction. This prove that $x(t)$ is extensible to $[-\tau,+\infty)$, with $|x(t)| \leq\|\varphi\|$ for all $t>0$.

As a finally notation, for a function $P: X \rightarrow X$ and $k \in \mathbb{N}$, we denote the composition $\underbrace{P \circ \cdots \circ P}_{k \text { times }}$ by $P^{k}$. 


\section{Global exponential stability}

In the phase space $C_{n}$, consider the following nonautonomous system of delayed differential equations

$$
x_{i}^{\prime}(t)=-\rho_{i}\left(t, x_{t}\right)\left[b_{i}\left(t, x_{i}(t)\right)+f_{i}\left(t, x_{t}\right)\right], \quad t \geq 0, i=1, \ldots, n,
$$

where $\rho_{i}:[0,+\infty) \times C_{n} \rightarrow \mathbb{R}^{+}, f_{i}:[0,+\infty) \times C_{n} \rightarrow \mathbb{R}$, and $b_{i}:[0,+\infty) \times \mathbb{R} \rightarrow \mathbb{R}$ are continuous functions. This model is a small generalization of a model introduced in [7] and it is particularly relevant in terms of applications, since it includes different types of neural network models with delays, such as Hopfield, Cohen-Grossberg, and BAM.

For (3.1) the following hypotheses will be considered:

(A1) there exists a $x^{*}=\left(x_{1}^{*}, \ldots, x_{n}^{*}\right) \in \mathbb{R}^{n}$ equilibrium point of (3.1);

(A2) for each $i \in\{1, \ldots, n\}$, there exists a $\underline{\rho}_{i}>0$ such that

$$
\underline{\rho}_{i}=\inf \left\{\rho_{i}(t, \varphi): t \geq 0, \varphi \in C_{n}\right\} ;
$$

(A3) for each $i \in\{1, \ldots, n\}$, there exists a function $\beta_{i}:[0,+\infty) \rightarrow \mathbb{R}^{+}$such that

$$
\left(b_{i}(t, u)-b_{i}(t, v)\right) /(u-v) \geq \beta_{i}(t)>0, \quad \forall t \geq 0, \forall u, v \in \mathbb{R}, u \neq v
$$

(A4) for each $i \in\{1, \ldots, n\}, f_{i}:[0,+\infty) \times C_{n} \rightarrow \mathbb{R}$ is a Lipschitz function on the second variable that is, there exists a function $l_{i}:[0,+\infty) \rightarrow \mathbb{R}^{+}$such that

$$
\left|f_{i}(t, \varphi)-f_{i}(t, \psi)\right| \leq l_{i}(t)\|\varphi-\psi\|, \quad \forall t \geq 0, \forall \varphi, \psi \in C_{n}
$$

(A5) there exist $\varepsilon>0$ and a continuous function $\lambda:[-\tau,+\infty) \rightarrow \mathbb{R}^{+}$such that, for each $i \in\{1, \ldots, n\}$,

$$
\underline{\rho}_{i}\left(\beta_{i}(t)-l_{i}(t) e^{\int_{t-\tau}^{t} \lambda(s) d s}\right)>\lambda(t) \quad \text { and } \quad \int_{0}^{t} \lambda(s) d s \geq \varepsilon t, \text { for all } t \geq 0 .
$$

In the following Lemma, we show that the solutions of (3.1) are defined and bounded on $[-\tau,+\infty)$.

Lemma 3.1. For (3.1) assume hypotheses (A1), (A3), and (A4), and suppose that

$$
\beta_{i}(t)-l_{i}(t)>0 \quad \text { for all } t \geq 0, \text { and } i=1, \ldots, n \text {. }
$$

Then, each solution $x(t)=x(t, 0, \varphi)$ (with $\varphi \in C_{n}$ ) of (3.1) is defined and bounded on $[0,+\infty)$ and it satisfies $\left|x(t)-x^{*}\right| \leq\left\|\varphi-x^{*}\right\|$ for all $t \geq 0$.

Proof. Let $x^{*}=\left(x_{1}^{*}, \ldots, x_{n}^{*}\right) \in \mathbb{R}^{n}$ be an equilibrium point of $(3.1)$, that is,

$$
b_{i}\left(t, x_{i}^{*}\right)+f_{i}\left(t, x^{*}\right)=0, \quad \text { for all } t \geq 0, i=1, \ldots, n .
$$

By the translation $\bar{x}(t)=x(t)-x^{*}$, the system (3.1) has the form

$$
\bar{x}_{i}^{\prime}(t)=-\rho_{i}\left(t, \bar{x}_{t}+x^{*}\right)\left[b_{i}\left(t, \bar{x}_{i}(t)+x_{i}^{*}\right)+f_{i}\left(t, \bar{x}_{t}+x^{*}\right)\right], \quad t \geq 0, i=1, \ldots, n .
$$


Clearly, (3.4) has the form (3.1), for which zero is an equilibrium point, and hypotheses (A3) and (A4) hold with the same functions $\beta_{i}(t)$ and $l_{i}(t)$. Hence, without loss of generality, we may consider $x^{*}=0$ in the system (3.1).

Take $t \geq 0$ and $\varphi \in C_{n}$ such that $|\varphi(\theta)|<|\varphi(0)|$ for $\theta \in[-\tau, 0)$. Let $i$ be such that $\|\varphi\|=\left|\varphi_{i}(0)\right|$ and suppose that $\varphi_{i}(0)>0$ (the case $\varphi_{i}(0)<0$ is analogous). Then,

$$
\begin{aligned}
b_{i}\left(t, \varphi_{i}(0)\right)+f_{i}(t, \varphi) & =\left[b_{i}\left(t, \varphi_{i}(0)\right)-b_{i}(t, 0)\right]+\left[f_{i}(t, \varphi)-f_{i}(t, 0)\right] \\
& \geq \beta_{i}(t) \varphi_{i}(0)-l_{i}(t)\|\varphi\|=\beta_{i}(t) \varphi_{i}(0)-l_{i}(t) \varphi_{i}(0) \\
& =\left(\beta_{i}(t)-l_{i}(t)\right) \varphi_{i}(0)>0 .
\end{aligned}
$$

This proves that $\mathbf{( H )}$ holds and the result follows from Lemma 2.1.

Now, we state the main result on the global exponential stability of the equilibrium point $x^{*}$ of $(3.1)$.

Theorem 3.1. Consider the FDE (3.1) under the hypotheses (A1)-(A5).

Then, the equilibrium point of (3.1) is globally exponentially stable.

Proof. As in proof of Lemma 3.1, by translation, we may assume that zero is an equilibrium point, which means, $b_{i}(t, 0)+f_{i}(t, 0)=0$ for all $t \geq 0$ and $i=1, \ldots, n$.

Since the hypothesis (A5) implies (3.3), then, from Lemma 3.1, we deduce that all solutions are defined and bounded on $[0,+\infty)$, and $x=0$ is uniformly stable.

As $\lambda(t)>0$ for all $t \geq 0$, the change of variables $z(t)=e^{\int_{0}^{t} \lambda(s) d s} x(t)$ transforms the system (3.1) into

$$
z_{i}^{\prime}(t)=g_{i}\left(t, z_{t}\right), \quad t \geq 0, i=1, \ldots, n,
$$

where,

$g_{i}(t, \varphi)=\lambda(t) \varphi_{i}(0)-\rho_{i}\left(t, e^{-\int_{0}^{t+\cdot} \lambda(s) d s} \varphi\right) e^{\int_{0}^{t} \lambda(s) d s}\left[b_{i}\left(t, e^{-\int_{0}^{t} \lambda(s) d s} \varphi_{i}(0)\right)+f_{i}\left(t, e^{-\int_{0}^{t+\cdot} \lambda(s) d s} \varphi\right)\right]$

Now, take $t \geq 0$ and $\varphi \in C_{n}$ such that $|\varphi(\theta)|<|\varphi(0)|$ for $\theta \in[-\tau, 0)$. Let $i$ be such that $\|\varphi\|=\left|\varphi_{i}(0)\right|$ and suppose that $\varphi_{i}(0)>0$ (the case $\varphi_{i}(0)<0$ is analogous). Then

$$
\begin{aligned}
g_{i}(t, \varphi)= & \lambda(t) \varphi_{i}(0)-\rho_{i}\left(t, e^{-\int_{0}^{t+\cdot} \lambda(s) d s} \varphi\right) e^{\int_{0}^{t} \lambda(s) d s}\left[b_{i}\left(t, e^{-\int_{0}^{t} \lambda(s) d s} \varphi_{i}(0)\right)-b_{i}(t, 0)\right. \\
& \left.+f_{i}\left(t, e^{-\int_{0}^{t+\cdot} \lambda(s) d s} \varphi\right)-f_{i}(t, 0)\right] \\
\leq & \lambda(t) \varphi_{i}(0)-\rho_{i}\left(t, e^{-\int_{0}^{t+\cdot} \lambda(s) d s} \varphi\right) e^{\int_{0}^{t} \lambda(s) d s}\left[\beta_{i}(t) e^{-\int_{0}^{t} \lambda(s) d s} \varphi_{i}(0)-l_{i}(t) e^{-\int_{0}^{t-\tau} \lambda(s) d s}\|\varphi\|\right] \\
\leq & \varphi_{i}(0)\left[\lambda(t)-\underline{\rho}_{i}\left(\beta_{i}(t)-l_{i}(t) e^{\int_{t-\tau}^{t} \lambda(s) d s}\right)\right],
\end{aligned}
$$

and, from the hypothesis (A5), we get $g_{i}(t, \varphi)<0$ and the hypothesis $(\mathbf{H})$ holds. Consequently, from Lemma 2.1, we deduce that the solution $z(t)$ of (3.5) satisfies $|z(t)| \leq\left\|z_{0}\right\|$ for all $t \geq 0$. Thus, again from the hypothesis (A5), we obtain

$$
|x(t, 0, \varphi)|=\left|e^{-\int_{0}^{t} \lambda(s) d s} z\left(t, 0, e^{\int_{0}^{\cdot} \lambda(s) d s} \varphi\right)\right| \leq e^{-\varepsilon t}\left|z\left(t, 0, e^{-\int^{0} \lambda(s) d s} \varphi\right)\right| \leq e^{-\varepsilon t}\|\varphi\| \quad \forall t \geq 0,
$$

and we have the result. 
Corollary 3.1. Assume that hypotheses (A1)-(A4) are satisfied.

If the functions $l_{i}(t)$ are bounded and there exists $\alpha>0$ such that

$$
\beta_{i}(t)-l_{i}(t)>\alpha, \quad \text { for all } t \geq 0, i=1, \ldots, n,
$$

then the equilibrium point of (3.1) is globally exponentially stable.

Proof. As a consequence of the Theorem 3.1, we just need to find a function $\lambda:[-\tau,+\infty) \rightarrow$ $\mathbb{R}^{+}$such that (3.2) holds. As $l_{i}(t)$ are bounded functions, there is $L>0$ such that $l_{i}(t)<L$ for all $t \geq 0$ and $i=1, \ldots, n$ and from (3.7) we conclude that

$$
\beta_{i}(t)-l_{i}(t)\left(1+\frac{\alpha}{2 L}\right)>\frac{\alpha}{2}, \quad t \geq 0, i=1, \ldots, n,
$$

and consequently, for some $d<0$, we have

$$
-\underline{\rho}_{i}\left(\beta_{i}(t)-l_{i}(t)\left(1+\frac{\alpha}{2 L}\right)\right)<d<0, \quad t \geq 0, i=1, \ldots, n .
$$

Now, considering $\varepsilon^{*}=\frac{1}{\tau} \log \left(1+\frac{\alpha}{2 L}\right)>0$ and $\varepsilon=\min \left\{-d, \varepsilon^{*}\right\}$, we conclude that

$$
\varepsilon-\underline{\rho}_{i}\left(\beta_{i}(t)-l_{i}(t) e^{\tau \varepsilon}\right)<0, \quad t \geq 0, i=1, \ldots, n,
$$

and (3.2) holds taking $\lambda(t)=\varepsilon$, for all $t \in[-\tau,+\infty)$.

Now, considering in (3.1) $\rho_{i} \equiv 1$ for all $i=1 \ldots, n$, we get the following systems of FDEs

$$
x_{i}^{\prime}(t)=-b_{i}\left(t, x_{i}(t)\right)+f_{i}\left(t, x_{t}\right), \quad t \geq 0, i=1, \ldots, n,
$$

where $b_{i}:[0,+\infty) \times \mathbb{R} \rightarrow \mathbb{R}$ and $f_{i}:[0+\infty) \times C_{n} \rightarrow \mathbb{R}$ are continuous. The next result establishes the global exponential stability of (3.10).

Corollary 3.2. Assume that hypotheses (A3)-(A5) are satisfied.

Then the system (3.10) is globally exponentially stable.

Proof. Let $\bar{x}(t)=x(t, 0, \bar{\varphi})$ be the solution of (3.10) with $\bar{\varphi} \in C_{n}$. The change of variables $z(t)=x(t)-\bar{x}(t)$ transforms the system (3.10) into

$$
z_{i}^{\prime}(t)=-b_{i}\left(t, z_{i}(t)+\bar{x}_{i}(t)\right)+f_{i}\left(t, z_{t}+\bar{x}_{t}\right)+b_{i}\left(t, \bar{x}_{i}(t)\right)-f_{i}\left(t, \bar{x}_{t}\right), \quad t \geq 0, i=1, \ldots,
$$

which can be written in the form

$$
z_{i}^{\prime}(t)=-\bar{b}_{i}\left(t, z_{i}(t)\right)+\bar{f}_{i}\left(t, z_{t}\right) \quad t \geq 0, i=1, \ldots, n,
$$

with $\bar{b}_{i}(t, u)=b_{i}\left(t, u+\bar{x}_{i}(t)\right)$ and $\bar{f}_{i}(t, \varphi)=f_{i}\left(t, \varphi+\bar{x}_{t}\right)+b_{i}\left(t, \bar{x}_{i}(t)\right)-f_{i}\left(t, \bar{x}_{t}\right)$ for $u \in \mathbb{R}$, $t \geq 0$, and $\varphi \in C_{n}$. It is easy to see that zero is an equilibrium point of (3.12), $\bar{b}_{i}$ satisfy (A3) with the same functions $\beta_{i}(t)$, and, for all $t \geq 0, \varphi, \psi \in C_{n}$, we have

$$
\begin{aligned}
\left|\bar{f}_{i}(t, \varphi)-\bar{f}_{i}(t, \psi)\right| & =\left|f_{i}\left(t, \varphi+\bar{x}_{t}\right)+b_{i}\left(t, \bar{x}_{i}(t)\right)-f_{i}\left(t, \bar{x}_{t}\right)-f_{i}\left(t, \psi+\bar{x}_{t}\right)-b_{i}\left(t, \bar{x}_{i}(t)\right)+f_{i}\left(t, \bar{x}_{t}\right)\right| \\
& =\left|f_{i}\left(t, \varphi+\bar{x}_{t}\right)-f_{i}\left(t, \psi+\bar{x}_{t}\right)\right| \\
& \leq l_{i}(t)\|\varphi-\psi\|
\end{aligned}
$$

which implies that (A4) holds with the same functions $l_{i}(t)$. Consequently, from Theorem 3.1 , we conclude that $|z(t)| \leq e^{-\varepsilon t}\left\|z_{0}\right\|$ which means that

$$
|x(t)-\bar{x}(t)| \leq e^{-\varepsilon t}\|\varphi-\bar{\varphi}\| \quad \forall t \geq 0,
$$

where $\varphi:=x_{0}$. As the constant $\varepsilon$ in the hypothesis (A5) is independent of $\bar{x}(t)$, we conclude that the system (3.10) is globally exponentially stable. 
In applications, nonautonomous neural network models with distributed delays often take the form

$$
x_{i}^{\prime}(t)=-\rho_{i}\left(t, x_{t}\right)\left[b_{i}\left(t, x_{i}(t)\right)+\sum_{k=1}^{K} \sum_{j=1}^{n} g_{i j k}\left(t, x_{j_{t}}\right)\right], \quad t \geq 0, i=1, \ldots, n,
$$

where, for each $i, j=1, \ldots, n$ and $k=1, \ldots, K, \tau_{i j k} \in[0, \tau]$ and $g_{i j k}:[0,+\infty) \times C\left(\left[-\tau_{i j k}, 0\right] ; \mathbb{R}\right) \rightarrow$ $\mathbb{R}$ is a continuous function satisfying the Lipschitz condition

$$
\left|g_{i j k}(t, \varphi)-g_{i j k}(t, \psi)\right| \leq l_{i j k}(t)\|\varphi-\psi\|, \quad \forall t \geq 0, \forall \varphi, \psi \in C\left(\left[-\tau_{i j k}, 0\right] ; \mathbb{R}\right),
$$

for some function $l_{i j k}:[0,+\infty) \rightarrow \mathbb{R}^{+}$. In what follows, for each $\varphi \in C([-\tau, 0] ; \mathbb{R})$, we denote

$$
g_{i j k}(t, \varphi):=g_{i j k}\left(t, \varphi_{\left.\right|_{\left[-\tau_{i j k}, 0\right]}}\right), \quad \text { for all } t \geq 0, i, j=1, \ldots, n, k=1, \ldots, K .
$$

For model (3.14), instead of (A5) assume the following hypothesis:

(A5') there exist $\varepsilon>0$ and a continuous function $\lambda: \mathbb{R} \rightarrow \mathbb{R}^{+}$such that, for each $i \in$ $\{1, \ldots, n\}$,

$$
\underline{\rho}_{i}\left(\beta_{i}(t)-\sum_{k=1}^{K} \sum_{j=1}^{n} l_{i j k}(t) e^{\int_{t-\tau_{i j k}}^{t} \lambda(s) d s}\right)>\lambda(t) \quad \text { and } \quad \int_{0}^{t} \lambda(s) d s \geq \varepsilon t, \forall t \geq 0
$$

In the following result, a slight improvement of Theorem 3.1 is given when (3.1) has the form (3.14). In the proof, the same ideas are used.

Theorem 3.2. Consider the FDE (3.14) assuming the hypotheses (A1)-(A3), (A5'), and (3.15).

Then, the equilibrium point of (3.14) is globally exponentially stable.

Proof. By translation, we may assume that zero is an equilibrium point that is,

$$
b_{i}(t, 0)+\sum_{k=1}^{K} \sum_{j=1}^{n} g_{i j k}(t, 0)=0, \quad t \geq 0, i=1, \ldots, n .
$$

The hypothesis (A5') implies $\beta_{i}(t)-\sum_{k=1}^{K} \sum_{j=1}^{n} l_{i j k}(t)>0$ and, since $\sum_{k=1}^{K} \sum_{j=1}^{n} l_{i j k}(t)$ is the Lipschitz constant with respect to the second argument of

$$
f_{i}(t, \varphi):=\sum_{k=1}^{K} \sum_{j=1}^{n} g_{i j k}\left(t, \varphi_{j}\right), \quad t \geq 0, \varphi=\left(\varphi_{1}, \ldots, \varphi_{n}\right) \in C_{n},
$$

then, from Lemma 3.1, we deduce that all solutions are defined and bounded on $[0,+\infty)$, and that $x=0$ is uniformly stable.

As $\lambda(t)>0$ for all $t \geq 0$, the change of variables $z(t)=e^{\int_{0}^{t} \lambda(s) d s} x(t)$ transforms the system (3.14) into

$$
z_{i}^{\prime}(t)=g_{i}\left(t, z_{t}\right) \quad t \geq 0, i=1, \ldots, n
$$


where, for all $t \geq 0$ and $i=1, \ldots, n$,

$$
\begin{array}{r}
g_{i}(t, \varphi)=\lambda(t) \varphi_{i}(0)-\rho_{i}\left(t, e^{-\int_{0}^{t+\cdot} \lambda(s) d s} \varphi\right) e^{\int_{0}^{t} \lambda(s) d s}\left[b_{i}\left(t, e^{-\int_{0}^{t} \lambda(s) d s} \varphi_{i}(0)\right)\right. \\
\left.+\sum_{k=1}^{K} \sum_{j=1}^{n} g_{i j k}\left(t, e^{-\int_{0}^{t+\cdot} \lambda(s) d s} \varphi_{j}\right)\right] .
\end{array}
$$

Now, take $t \geq 0$ and $\varphi \in C_{n}$ with $|\varphi(\theta)|<|\varphi(0)|$ for $\theta \in[-\tau, 0)$. Let $i$ be such that $\|\varphi\|=\left|\varphi_{i}(0)\right|$ and suppose that $\varphi_{i}(0)>0$ (the case $\varphi_{i}(0)<0$ is analogous). Then, from the hypotheses, we get

$$
\begin{aligned}
g_{i}(t, \varphi)= & \lambda(t) \varphi_{i}(0)-\rho_{i}\left(t, e^{-\int_{0}^{t+\cdot} \lambda(s) d s} \varphi\right) e^{\int_{0}^{t} \lambda(s) d s}\left[b_{i}\left(t, e^{-\int_{0}^{t} \lambda(s) d s} \varphi_{i}(0)\right)-b_{i}(t, 0)\right. \\
& \left.+\sum_{k=1}^{K} \sum_{j=1}^{n} g_{i j k}\left(t, e^{-\int_{0}^{t+\cdot} \lambda(s) d s} \varphi_{j}\right)-\sum_{k=1}^{K} \sum_{j=1}^{n} g_{i j k}(t, 0)\right] \\
\leq & \lambda(t) \varphi_{i}(0)-\rho_{i}\left(t, e^{-\int_{0}^{t+\cdot} \lambda(s) d s} \varphi\right) e^{\int_{0}^{t} \lambda(s) d s}\left[\beta_{i}(t) e^{-\int_{0}^{t} \lambda(s) d s} \varphi_{i}(0)\right. \\
& \left.-\sum_{k=1}^{K} \sum_{j=1}^{n} l_{i j k}(t) e^{-\int_{0}^{t-\tau_{i j k}} \lambda(s) d s}\left\|\varphi_{j}\right\|\right] \\
\leq & \varphi_{i}(0)\left[\lambda(t)-\underline{\rho}_{i}\left(\beta_{i}(t)-\sum_{k=1}^{K} \sum_{j=1}^{n} l_{i j k}(t) e^{-\int_{t}^{t-\tau_{i j k}} \lambda(s) d s}\right)\right]
\end{aligned}
$$

and, from the hypothesis (A5'), we conclude that $g_{i}(t, \varphi)<0$ and the hypothesis $(\mathbf{H})$ holds. Consequently, from Lemma 2.1, we deduce that the solutions $z(t)$ of (3.17) satisfy $|z(t)| \leq\left\|z_{0}\right\|$ for all $t \geq 0$. Thus, again from the hypothesis (A5'), we obtain

$$
|x(t, 0, \varphi)|=\left|e^{-\int_{0}^{t} \lambda(s) d s} z\left(t, 0, e^{\int_{0} \lambda(s) d s} \varphi\right)\right| \leq e^{-\varepsilon t}\left|z\left(t, 0, e^{-\int^{0} \lambda(s) d s} \varphi\right)\right| \leq e^{-\varepsilon t}\|\varphi\| \quad \forall t \geq 0,
$$

and we have the result.

Following the same ideas presented in the proof of Corollary 3.1, we can obtain the following result.

Corollary 3.3. Consider the FDE (3.14) assuming the hypotheses (A1)-(A3), and (3.15).

If the functions $l_{i j k}(t)$ are bounded and there exists $\alpha>0$ such that

$$
\beta_{i}(t)-\sum_{k=1}^{K} \sum_{j=1}^{n} l_{i j k}(t)>\alpha, \quad \text { for all } t \geq 0, i=1, \ldots, n,
$$

then the equilibrium point of (3.14) is globally exponentially stable.

Following the same ideas presented in the proof of Corollary 3.2, we also can obtain the following result.

Corollary 3.4. Assume that (A3), (A5'), and (3.15) are satisfied.

Then the system

$$
x_{i}^{\prime}(t)=-b_{i}\left(t, x_{i}(t)\right)+\sum_{k=1}^{K} \sum_{j=1}^{n} g_{i j k}\left(t, x_{j_{t}}\right), \quad t \geq 0, i=1, \ldots, n,
$$

is globally exponentially stable. 


\section{Existence and exponential stability of periodic solution}

In this section, we apply the contraction mapping principle to derive a criterion to ensure that system (3.10) has a unique periodic solution and all other solutions converge to it with exponential rates.

Here, we take $\omega>0$ and, in the phase space $C_{n}$, we consider the FDE (3.10) where the continuous functions $b_{i}:[0,+\infty) \times \mathbb{R} \rightarrow \mathbb{R}$ and $f_{i}:[0,+\infty) \times C_{n} \rightarrow \mathbb{R}$ are $\omega$-periodic with respect to the first argument, that is,

$$
b_{i}(t, u)=b_{i}(t+\omega, u), \quad \forall t \geq 0, \quad \forall u \in \mathbb{R},
$$

and

$$
f_{i}(t, \varphi)=f_{i}(t+\omega, \varphi), \quad \forall t \geq 0, \quad \forall \varphi \in C_{n} .
$$

Theorem 4.1. Assume that hypotheses (A3) and (A4) are satisfied.

If there is $\alpha>0$ such that

$$
\beta_{i}(t)-l_{i}(t)>\alpha, \quad \forall t \in[0, \omega],
$$

then the system (3.10) has a unique $\omega$-periodic solution which is globally exponentially stable.

Proof. As $b_{i}(t, u)$ are continuous and $\omega$-periodic with respect to the first argument then, from (A3), $\beta_{i}(t)$ are bounded and consequently $l_{i}(t)$ are also bounded. Thus, as in the proof of Corollary 3.1, the hypothesis (A5) holds and, from Corollary 3.2, we conclude that there is $\varepsilon>0$ such that

$$
|x(t, 0, \varphi)-x(t, 0, \bar{\varphi})| \leq e^{-\varepsilon t}\|\varphi-\bar{\varphi}\|, \quad \forall t \geq 0, \forall \varphi, \bar{\varphi} \in C_{n},
$$

and consequently

$$
\left\|x_{t}(\varphi)-x_{t}(\bar{\varphi})\right\| \leq e^{-\varepsilon(t-\tau)}\|\varphi-\bar{\varphi}\|, \quad \forall t \geq \tau, \forall \varphi, \bar{\varphi} \in C_{n} .
$$

Now, we can choose $k \in \mathbb{N}$ such that

$$
k \omega>\tau \quad \text { and } \quad e^{-(k \omega-\tau)} \leq \frac{1}{2},
$$

and we define the map $P: C_{n} \rightarrow C_{n}$ by $P(\varphi)=x_{\omega}(\varphi)$. For $\varphi, \bar{\varphi} \in C_{n}$, from (4.3) and (4.4) we have

$$
\begin{aligned}
\left\|P^{k}(\varphi)-P^{k}(\bar{\varphi})\right\| & =\left\|P\left(P^{k-1}(\varphi)\right)-P\left(P^{k-1}(\bar{\varphi})\right)\right\|=\left\|x_{\omega}\left(P^{k-1}(\varphi)\right)-x_{\omega}\left(P^{k-1}(\bar{\varphi})\right)\right\| \\
& =\left\|x_{\omega}\left(x_{\omega}\left(P^{k-2}(\varphi)\right)\right)-x_{\omega}\left(x_{\omega}\left(P^{k-2}(\bar{\varphi})\right)\right)\right\|=\left\|x_{k \omega}(\varphi)-x_{k \omega}(\bar{\varphi})\right\| \\
& \leq e^{-\varepsilon(k \omega-\tau)}\|\varphi-\bar{\varphi}\| \leq \frac{1}{2}\|\varphi-\bar{\varphi}\|,
\end{aligned}
$$

which implies that $P^{k}$ is a contraction map on $C_{n}$. As $C_{n}$ is a Banach space, we conclude that there is a unique fixed point $\varphi^{*} \in C_{n}$ such that $P^{k}\left(\varphi^{*}\right)=\varphi^{*}$. Noting that

$$
P^{k}\left(P\left(\varphi^{*}\right)\right)=P\left(P^{k}\left(\varphi^{*}\right)\right)=P\left(\varphi^{*}\right),
$$

we have $P\left(\varphi^{*}\right)=\varphi^{*}$ which means that $x_{\omega}\left(\varphi^{*}\right)=\varphi^{*}$. 
Finally, as $x\left(t, 0, \varphi^{*}\right)$ is a solution of $(3.10)$ and $b_{i}(t, u)$ and $f_{i}(t, \varphi)$ are $\omega$-periodic with respect to the first argument, we know that $x\left(t+\omega, 0, \varphi^{*}\right)$ is also a solution of (3.10) and

$$
x\left(t, 0, \varphi^{*}\right)=x\left(t, 0, x_{\omega}\left(\varphi^{*}\right)\right)=x\left(t+\omega, 0, \varphi^{*}\right), \quad \forall t \geq 0 .
$$

Therefore $x\left(t, 0, \varphi^{*}\right)$ is the $\omega$-periodic solution of (3.10) and, from (4.2), all other solutions converge to it with exponential rates.

Remark 4.1 Observe that, in this setting, (A5) is equivalent to (4.1). In fact, on the one hand, as $b_{i}(t, u)$ are continuous and $\omega$-periodic with respect to the first argument, we conclude that $\beta_{i}(t)$ are bounded and, from $(4.1), l_{i}(t)$ are also bounded. Now, from Corollary 3.1 we conclude that (4.1) implies (A5). On the other hand, as $\beta_{i}(t)$ and $l_{i}(t)$ are $\omega$-periodic and $\lambda(t)$ is continuous on the compact set $[0, \omega]$, it is easy to conclude that (3.2) implies (4.1), where $\alpha:=\min _{t \in[0, \omega]} \lambda(t)$.

\section{Applications}

Here, we consider the following generalized nonautonomous Hopfield neural network model with continuous distributed time varying delays,

$x_{i}^{\prime}(t)=-b_{i}\left(t, x_{i}(t)\right)+\sum_{k=1}^{K} \sum_{j=1}^{n} f_{i j k}\left(t, \int_{-\tau_{i j k}}^{0} h_{i j k}\left(x_{j}(t+s)\right) d \eta_{i j}^{(k)}(t, s)\right), \quad t \geq 0, i=1, \ldots, n$

where, for $i, j=1, \ldots, n$ and $k=1, \ldots, K, \tau_{i j k}$ are nonnegative numbers, $b_{i}, f_{i j k}:[0,+\infty) \times$ $\mathbb{R} \rightarrow \mathbb{R}, \quad h_{i j k}: \mathbb{R} \rightarrow \mathbb{R}$, are continuous functions, and $\eta_{i j}^{(k)}:[0,+\infty) \times\left[-\tau_{i j k}, 0\right] \rightarrow \mathbb{R}$ are functions such that $t \mapsto \int_{-\tau_{i j k}}^{0} \psi(s) d \eta_{i j}^{(k)}(t, s)$ are continuous real functions, for all $\psi \in C\left(\left[-\tau_{i j k}, 0\right] ; \mathbb{R}\right)$, and $\eta_{i j}^{(k)}(t, \cdot)$ are non-decreasing and normalized, that is $\eta_{i j}^{(k)}(t, 0)-$ $\eta_{i j}^{(k)}\left(t,-\tau_{i j k}\right)=1$, for all $t \geq 0$.

Theorem 5.1. Consider (5.1) where $h_{i j k}$ are Lipschitz functions with Lipschitz constant $\gamma_{i j k}$ and $f_{i j k}$ are Lipschitz functions on the second variable, with Lipschitz constants $\mu_{i j k}(t)$, for $i, j=1, \ldots n$ and $k=1, \ldots, K$. Assume in addition that,

(i) (A3) holds;

(ii) There exist $d=\left(d_{1}, \ldots, d_{n}\right)>0, \varepsilon>0$, and $\lambda: \mathbb{R} \rightarrow \mathbb{R}^{+}$continuous such that

$$
\beta_{i}(t)-\sum_{k=1}^{K} \sum_{j=1}^{n} d_{i}^{-1} d_{j} \gamma_{i j k} \mu_{i j k}(t) e^{\int_{t-\tau_{i j k}}^{t} \lambda(s) d s}>\lambda(t), \quad \text { and } \quad \int_{0}^{t} \lambda(s) d s \geq \varepsilon t, \forall t \geq 0 .
$$

Then, the system (5.1) is globally exponentially stable.

Proof. The change $y_{i}(t)=d_{i}^{-1} x_{i}(t)$ transforms (5.1) into

$$
y_{i}^{\prime}(t)=-d_{i}^{-1} b_{i}\left(t, d_{i} y_{i}(t)\right)+\sum_{k=1}^{K} \sum_{j=1}^{n} d_{i}^{-1} f_{i j k}\left(t, \int_{-\tau_{i j p}}^{0} h_{i j k}\left(d_{j} y_{j}(t+s)\right) d \eta_{i j}^{(k)}(t, s)\right),
$$


which has the form (3.19) with, for each $t \geq 0, i, j=1, \ldots, n, k=1, \ldots, K$, and $\psi \in$ $C([-\tau, 0] ; \mathbb{R})$,

$$
g_{i j k}(t, \psi):=d_{i}^{-1} f_{i j k}\left(t, \int_{-\tau_{i j k}}^{0} h_{i j k}\left(d_{j} \psi(s)\right) d \eta_{i j}^{(k)}(t, s)\right) .
$$

Note that $\left(d_{i}^{-1} b_{i}\left(t, d_{i} u\right)-d_{i}^{-1} b_{i}\left(t, d_{i} v\right)\right) /(u-v)=\left(b_{i}\left(t, d_{i} u\right)-b_{i}\left(t, d_{i} v\right)\right) /\left(d_{i} u-d_{i} v\right) \geq$ $\beta_{i}(t)$ for all $t \geq 0$, and $u, v \in \mathbb{R}, u \neq v$, i.e., condition (A3) is satisfied. As $\eta_{i j}^{(k)}(t, \cdot)$ are non-decreasing and normalized functions, for each $i, j=1, \ldots, n, k=1, \ldots, K$, and $\varphi, \psi \in C\left(\left[-\tau_{i j k}, 0\right] ; \mathbb{R}\right)$, we have

$$
\begin{aligned}
\left|g_{i j k}(t, \varphi)-g_{i j k}(t, \psi)\right| & \leq d_{i}^{-1} \mu_{i j k}(t)\left|\int_{-\tau_{i j k}}^{0} h_{i j k}\left(d_{j} \varphi(s)\right) d \eta_{i j}^{(k)}(t, s)-\int_{-\tau_{i j k}}^{0} h_{i j k}\left(d_{j} \psi(s)\right) d \eta_{i j}^{(k)}(t, s)\right| \\
& \leq d_{i}^{-1} \mu_{i j k}(t) \int_{-\tau_{i j k}}^{0}\left|h_{i j k}\left(d_{j} \varphi(s)\right)-h_{i j k}\left(d_{j} \psi(s)\right)\right| d \eta_{i j}^{(k)}(t, s) \\
& \leq d_{i}^{-1} \mu_{i j k}(t) \gamma_{i j k} d_{j} \int_{-\tau_{i j k}}^{0}|\varphi(s)-\psi(s)| d \eta_{i j}^{(k)}(t, s) \\
& \leq\left(d_{i}^{-1} d_{j} \gamma_{i j k} \mu_{i j k}(t)\right)\|\varphi-\psi\| .
\end{aligned}
$$

Consequently, the condition (3.15) holds and, from the hypothesis (ii), the condition (A5') also holds. Now, the result follows from Corollary 3.4.

Example 5.1. If we take $K=2, b_{i}(t, u)=c_{i}(t) u, h_{i j k}(u)=u, f_{i j 1}(t, u)=a_{i j}(t) f_{j}(u)$, $f_{i j 2}(t, u)=b_{i j}(t) f_{j}(u)+\frac{I_{i}(t)}{n}, \eta_{i j}^{(1)}:[0,+\infty) \times\left[-\tau_{i j 1}, 0\right] \rightarrow \mathbb{R}$ defined by

$$
\eta_{i j}^{(1)}(t, s)=\eta_{i j}^{(1)}(s)=\left\{\begin{array}{l}
0, \quad-\tau_{i j 1} \leq s<0 \\
1, \quad s=0
\end{array},\right.
$$

and $\eta_{i j}^{(2)}:[0,+\infty) \times\left[-\tau_{i j 2}, 0\right] \rightarrow \mathbb{R}$ defined by

$$
\eta_{i j}^{(2)}(t, s)=\left\{\begin{array}{l}
0, \quad-\tau_{i j 2} \leq s<-\tau_{i j}(t) \\
1, \quad-\tau_{i j}(t) \leq s \leq 0
\end{array},\right.
$$

for $t \geq 0, i, j=1, \ldots, n, k=1,2$, and $u \in \mathbb{R}$, where $f_{j}: \mathbb{R} \rightarrow \mathbb{R}, c_{i}, a_{i j}, b_{i j}, I_{i}:[0,+\infty) \rightarrow \mathbb{R}$, and $\tau_{i j}:[0,+\infty) \rightarrow[0,+\infty)$ are continuous functions, the model (5.1) becomes the following Hopfield neural network model:

$$
\dot{x}_{i}(t)=-c_{i}(t) x_{i}(t)+\sum_{j=1}^{n} a_{i j}(t) f_{j}\left(x_{j}(t)\right)+\sum_{j=1}^{n} b_{i j}(t) f_{j}\left(x_{j}\left(t-\tau_{i j}(t)\right)\right)+I_{i}(t), \quad t \geq 0 .
$$

Applying Theorem 5.1 to model (5.3), we have the following result.

Corollary 5.1. Consider (5.3), where $\tau_{i j}(t)$ are bounded and continuous functions and $f_{j}$ are Lipschitz functions with Lipschitz constants $L_{j}$. 
If there exit $d=\left(d_{1}, \ldots, d_{n}\right)>0, \varepsilon>0$, and $\lambda: \mathbb{R} \rightarrow \mathbb{R}^{+}$continuous such that, for all $t \geq 0$ and $i=1, \ldots, n$,

$c_{i}(t)-\sum_{j=1}^{n} d_{i}^{-1} d_{j}\left|a_{i j}(t)\right| L_{j}-\sum_{j=1}^{n} d_{i}^{-1} d_{j}\left|b_{i j}(t)\right| L_{j} e^{\int_{t-\tau_{i j}}^{t} \lambda(s) d s}>\lambda(t), \quad$ and $\quad \int_{0}^{t} \lambda(s) d s \geq \varepsilon t$, with $\tau_{i j}:=\sup _{t} \tau_{i j}(t)$, then the system (5.3) is globally exponentially stable.

Remark 5.1. The particular model (5.3) was recently studied in several papers such as [21], [22], and [11]. By comparison, for example, we can apply Corollary 5.1 to conclude that the particular model

$$
\left\{\begin{array}{l}
x_{1}^{\prime}(t)=-2 x_{1}(t)+(\sin t) x_{2}(t-|\sin t|) \\
x_{2}^{\prime}(t)=-4 x_{2}(t)+(\sin t) x_{1}(t)
\end{array},\right.
$$

is globally exponentially stable. However the Theorem 2 in [21] cannot be applied to get the same conclusion and, consequently, our previous result presents a different criterion.

Moreover, it is relevant to observe that the model (5.1) is general enough to include, as particular situations, some BAM neural network models. The following example illustrates that fact.

Example 5.2. Consider the following nonautonomous BAM neural network model with discrete time-varying delays

$$
\left\{\begin{array}{l}
x_{i}^{\prime}(t)=-\widetilde{c}_{i}(t) x_{i}(t)+\sum_{j=1}^{m} a_{i j}(t) f_{j}\left(y_{j}(t)\right)+\sum_{j=1}^{m} b_{i j}(t) f_{j}\left(y_{j}\left(t-\tau_{i j}(t)\right)\right)+\widetilde{I}_{i}(t), \quad i=1, \ldots, n \\
y_{j}^{\prime}(t)=-c_{j}(t) y_{j}(t)+\sum_{i=1}^{n} \widetilde{a}_{j i}(t) \widetilde{f}_{i}\left(x_{i}(t)\right)+\sum_{i=1}^{n} \widetilde{b}_{j i}(t) \widetilde{f}_{i}\left(x_{i}\left(t-\widetilde{\tau}_{j i}(t)\right)\right)+I_{j}(t), \quad j=1, \ldots, m,
\end{array}\right.
$$

As in the above example, it is easy to see that (5.5) is a special case of model (5.1), thus from Theorem 5.1 we obtain the following result.

Corollary 5.2. Consider (5.5) where $\tau_{i j}(t), \widetilde{\tau}_{j i}(t)$ are bounded and continuous functions and $f_{j}, \widetilde{f}_{i}$, are Lipschitz functions with Lipschitz constant, $F_{j}, \widetilde{F}_{i}$ respectively, $i=1, \ldots, n$, $j=1, \ldots, m$.

If there exist $\widetilde{d}=\left(\widetilde{d}_{1}, \ldots, \widetilde{d}_{n}\right)>0, d=\left(d_{1}, \ldots, d_{m}\right)>0, \varepsilon>0$, and $\lambda: \mathbb{R} \rightarrow \mathbb{R}^{+}$ continuous such that, for all $t \geq 0, \int_{0}^{t} \lambda(s) d s \geq$ st and

$$
\begin{aligned}
& \widetilde{c}_{i}(t)-\sum_{j=1}^{m} \widetilde{d}_{i}^{-1} d_{j}\left(\left|a_{i j}(t)\right| F_{j}+\left|b_{i j}(t)\right| F_{j} e^{\int_{t-\tau_{i j}}^{t} \lambda(s) d s}\right)>\lambda(t), \quad i=1, \ldots, n, \\
& c_{j}(t)-\sum_{i=1}^{n} d_{j}^{-1} \widetilde{d}_{i}\left(\left|\widetilde{a}_{j i}(t)\right| \widetilde{F}_{i}+\left|\widetilde{b}_{j i}(t)\right| \widetilde{F}_{i} e^{\int_{t-\tau_{j i}}^{t} \lambda(s) d s}\right)>\lambda(t), \quad j=1, \ldots, m,
\end{aligned}
$$

with $\tau_{i j}:=\sup _{t} \tau_{i j}(t)$ and $\widetilde{\tau}_{j i}:=\sup _{t} \widetilde{\tau}_{j i}(t)$, then system (5.5) is globally exponentially stable. 
Now, we apply the results in section 4 to obtain sufficient criteria for the exponential stability of periodic neural network models.

Let $\omega>0$ and consider the system (5.1) where $t \mapsto \int_{-\tau_{i j k}}^{0} \psi(s) d \eta_{i j}^{(k)}(t, s)$ are $\omega$-periodic continuous real functions for all $\psi \in C\left(\left[-\tau_{i j k}, 0\right] ; \mathbb{R}\right)$, and the continuous functions $b_{i}, f_{i j k}$ : $[0,+\infty) \times \mathbb{R} \rightarrow \mathbb{R}$ are $\omega$-periodic with respect to the first argument, that is, $b_{i}(t, u)=$ $b_{i}(t+\omega, u)$ and $f_{i j k}(t, u)=f_{i j k}(t+\omega, u)$ for all $t \geq 0$ and $u \in \mathbb{R}$.

Theorem 5.2. Assume that (A3) holds and, for $i, j=1, \ldots, n, k=1, \ldots, K, h_{i j k}$ are Lipschitz functions with Lipschitz constant $\gamma_{i j k}, f_{i j k}$ are Lipschitz functions on the second variable with Lipschitz constant $\mu_{i j k}(t)$, and $\eta_{i j}^{(k)}(t, \cdot)$ are non-decreasing and normalized functions. If there exist $\alpha>0$ and $d=\left(d_{1}, \ldots, d_{n}\right)>0$ such that

$$
d_{i} \beta_{i}(t)-\sum_{j=1}^{n} d_{j} l_{i j}(t)>\alpha, \forall t \in[0, \omega], \forall i=1, \ldots, n
$$

with $l_{i j}(t):=\sum_{k=1}^{K} \mu_{i j k}(t) \gamma_{i j k}$, then the system (5.1) has a unique $\omega$-periodic solution which is globally exponentially stable.

Proof. The change $y_{i}(t)=d_{i}^{-1} x_{i}(t)$ transforms (5.1) into

$$
y_{i}^{\prime}(t)=-\bar{b}_{i}\left(t, y_{i}(t)\right)+\bar{f}_{i}\left(t, y_{t}\right), \quad i=1, \ldots, n, t \geq 0,
$$

where $\bar{b}_{i}(t, u):=d_{i}^{-1} b_{i}\left(t, d_{i} u\right)$ and

$$
\bar{f}_{i}(t, \varphi):=\sum_{j=1}^{n} \sum_{k=1}^{K} d_{i}^{-1} f_{i j k}\left(t, \int_{-\tau_{i j k}}^{0} h_{i j k}\left(d_{j} \varphi_{j}(s)\right) d \eta_{i j}^{(k)}(t, s)\right)
$$

are continuous and $\omega$-periodic functions with respect to the first argument. By one hand, as $h_{i j k}$ are Lipschitz functions, $f_{i j k}$ are Lipschitz functions on the second variable, and $\eta_{i j}^{(k)}(t, \cdot)$ are non-decreasing and normalized, then it easy to show that each $\bar{f}_{i}$ satisfies (A4) with

$$
l_{i}(t):=d_{i}^{-1} \sum_{j=1}^{n} d_{j} \sum_{k=1}^{K} \mu_{i j k}(t) \gamma_{i j k} .
$$

By other hand, as each $b_{i}$ satisfies condition (A3), then each $\bar{b}_{i}$ also satisfies condition (A3) with the same function $\beta_{i}(t)$.

Consequently, from (5.8), we have $d_{i} \beta_{i}(t)-d_{i} l_{i}(t)>\alpha$ which implies that

$$
\beta_{i}(t)-l_{i}(t)>\min _{j}\left\{\alpha d_{j}^{-1}\right\}>0, \quad \forall t \in[0, \omega], i=1, \ldots, n,
$$

and the result follows from Theorem 4.1.

Example 5.3. As we saw in the first example, the Hopfiel neural network model (5.3) is a particular case of model (5.1). Thus, applying Theorem 5.2 to model (5.3), we have the following result. 
Corollary 5.3. Consider (5.3), where $c_{i}, a_{i j}, \tau_{i j}, b_{i j}, I_{i}:[0,+\infty) \rightarrow \mathbb{R}$ are $\omega$-periodic continuous functions with $\tau_{i j}(t) \geq 0$, and $f_{j}$ are Lipschitz functions with Lipschitz constants $L_{j}$.

If there exists $d=\left(d_{1}, \ldots, d_{n}\right)>0$ such that

$$
d_{i} c_{i}(t)-\sum_{j=1}^{n} d_{j} L_{j}\left(\left|a_{i j}(t)\right|+\left|b_{i j}(t)\right|\right)>0, \quad \forall t \in[0, \omega], i=1, \ldots, n,
$$

then system (5.3) has a unique $\omega$-periodic solution which is globally exponentially stable.

Remark 5.2. In [17], the existence of a periodic solution and its global exponential stability of periodic system (5.3) were proved with the additional hypothesis:

$$
d_{i} c_{i}(t)-\sum_{j=1}^{n} d_{j} L_{i}\left(\left|a_{j i}(t)+\right| b_{j i} \mid\right)>0, \quad \forall t \in[0, \omega], i=1, \ldots, n .
$$

Hence, our Corollary 5.3 improves the main result in [17]. Moreover, as the model (5.3) is a particular case of system (5.1), then our Theorem 5.2 strongly improves the main result in $[17]$.

Example 5.4. In [20], the author studied the existence and global exponential stability of a unique $\omega$-periodic solution of the following $\omega$-periodic BAM neural network model with time-varying coefficients and distributed delays:

$$
\left\{\begin{aligned}
x_{i}^{\prime}(t)= & -\widetilde{c}_{i}(t) \widetilde{b}_{i}\left(x_{i}(t)\right)+\sum_{j=1}^{m} a_{i j}(t) f_{j}\left(y_{j}(t)\right) \\
& +\sum_{j=1}^{m} e_{i j}(t) \int_{-\tau}^{0} k_{i j}(-s) h_{j}\left(y_{j}\left(t-\tau_{i j}+s\right)\right) d s+\widetilde{I}_{i}(t), \quad i=1, \ldots, k, \\
y_{j}^{\prime}(t)=- & c_{j}(t) b_{j}\left(y_{j}(t)\right)+\sum_{i=1}^{k} \widetilde{a}_{j i}(t) \widetilde{f}_{i}\left(x_{i}(t)\right) \\
& +\sum_{i=1}^{k} \widetilde{e}_{j i}(t) \int_{-\sigma}^{0} \widetilde{k}_{j i}(-s) \widetilde{h}_{i}\left(x_{i}\left(t-\sigma_{j i}+s\right)\right) d s+I_{j}(t), \quad j=1, \ldots, m,
\end{aligned}\right.
$$

where $a_{i j}, \widetilde{a}_{j i}, \widetilde{I}_{i}, I_{j}:[0,+\infty) \rightarrow \mathbb{R}$ and $\widetilde{c}_{i}, c_{j}:[0,+\infty) \rightarrow(0,+\infty)$ are $\omega$-periodic continuous functions, $\widetilde{b}_{i}, b_{j}, f_{j}, \widetilde{f}_{i}, h_{j}, \widetilde{h}_{i}: \mathbb{R} \rightarrow \mathbb{R}$ are continuous functions, and $k_{i j}:[0, \tau] \rightarrow[0,+\infty)$, $\widetilde{k}_{j i}:[0, \sigma] \rightarrow[0,+\infty)$ are piecewise continuous functions, $i=1, \ldots, k, j=1, \ldots, m$.

System (5.11) is also a particular case of (5.1), when $n=k+m, K=2$,

$$
\begin{aligned}
& b_{i}(t, u)=\left\{\begin{array}{ll}
\widetilde{c}_{i}(t) \widetilde{b}_{i}(u), & i=1, \ldots, k \\
c_{i-k}(t) b_{i-k}(u), & i=k+1, \ldots, k+m
\end{array} \quad, \quad \forall t \geq 0, \forall u \in \mathbb{R},\right. \\
& f_{i j 1}(t, u)=\left\{\begin{array}{lll}
0, & i=1, \ldots, k, & j=1, \ldots, k \\
a_{i(j-k)}(t) f_{j-k}(u), & i=1, \ldots, k, & j=k+1, \ldots, k+m \\
\widetilde{a}_{(i-k) j}(t) \widetilde{f}_{j}(u), & i=k+1, \ldots, k+m, & j=1, \ldots, k \\
0, & i=k+1, \ldots, k+m, & j=k+1, \ldots, k+m
\end{array}, \quad \forall t \geq 0, \forall u \in\right.
\end{aligned}
$$




$$
f_{i j 2}(t, u)=\left\{\begin{array}{lll}
0, & i=1, \ldots, k, & j=1, \ldots, k \\
e_{i(j-k)}(t)\left(\int_{0}^{\tau} k_{i(j-k)}(s) d s\right) u+\frac{\widetilde{I}_{i}(t)}{m}, & i=1, \ldots, k, & j=k+1, \ldots, k+m \\
\widetilde{e}_{(i-k) j}(t)\left(\int_{0}^{\sigma} \widetilde{k}_{(i-k) j}(s) d s\right) u+\frac{I_{(i-k)}(t)}{k}, & i=k+1, \ldots, k+m, & j=1, \ldots, k \\
0, & i=k+1, \ldots, k+m, & j=k+1, \ldots, k+m
\end{array},\right.
$$

$\forall t \geq 0, \forall u \in \mathbb{R}$

$$
\begin{aligned}
& h_{i j 1}(u)=u, i, j=1, \ldots, k+m, \quad \forall u \in \mathbb{R},
\end{aligned}
$$

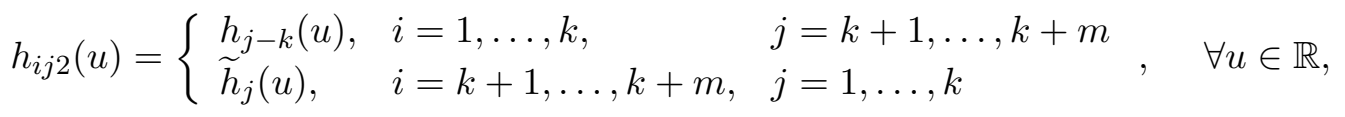

$$
\begin{aligned}
& \eta_{i j}^{(1)}(s)=\left\{\begin{array}{ll}
1, & s=0 \\
0, & s<0
\end{array},\right. \text { and } \\
& \eta_{i j}^{(2)}(s)=\left\{\begin{array}{lll}
\int_{-\tau-\tau_{i(j-k)}}^{s} k_{i(j-k)}^{*}(-v) d v, s \in\left[-\tau-\tau_{i(j-k)}, 0\right], & i=1, \ldots, k, & j=k+1, \ldots, k+m \\
\int_{-\sigma-\sigma_{(i-k) j}}^{s} \widetilde{k}_{(i-k) j}^{*}(-v) d v, s \in\left[-\sigma-\sigma_{(i-k) j}, 0\right], & i=k+1, \ldots, k+m, & j=1, \ldots, k
\end{array}\right.
\end{aligned}
$$

with, for $i=1, \ldots, k, j=1, \ldots, m, k_{i j}^{*}(t)=\left\{\begin{array}{ll}0, & t \in\left[0, \tau_{i j}\right) \\ \frac{k_{i j}\left(t-\tau_{i j}\right)}{\int_{0}^{\tau} k_{i j}(v) d v}, & t \in\left[\tau_{i j}, \tau_{i j}+\tau\right]\end{array}\right.$ and $\widetilde{k}_{j i}^{*}(t)=$

$$
\begin{cases}0, & t \in\left[0, \sigma_{j i}\right) \\ \frac{\widetilde{k}_{j i}\left(t-\sigma_{j i}\right)}{\int_{0}^{\sigma} \widetilde{k}_{j i}(v) d v}, & t \in\left[\sigma_{j i}, \sigma_{j i}+\sigma\right]\end{cases}
$$

Now, applying Theorem 5.2 to model (5.11), we have the following result.

Corollary 5.4. Consider (5.11) where $f_{j}, \widetilde{f}_{i}, h_{j}, \widetilde{h}_{i}$ are Lipschitz functions with Lipschitz constant, $F_{j}, \widetilde{F}_{i}, H_{j}, \widetilde{H}_{i}$ respectively, and there exist positive numbers $B_{j}, \widetilde{B}_{i}$ such that

$$
\left(\widetilde{b}_{i}(u)-\widetilde{b}_{i}(v)\right) /(u-v) \geq \widetilde{B}_{i},
$$

and

$$
\left(b_{j}(u)-b_{j}(v)\right) /(u-v) \geq B_{j},
$$

for all $u, v \in \mathbb{R}, u \neq v, i=1, \ldots, k, j=1, \ldots, m$.

If there exist $\widetilde{d}=\left(\widetilde{d}_{1}, \ldots, \widetilde{d}_{k}\right)>0$ and $d=\left(d_{1}, \ldots, d_{m}\right)>0$ such that, for all $t \in[0, \omega]$,

$$
\begin{gathered}
\widetilde{d}_{i} \widetilde{B}_{i} \widetilde{c}_{i}(t)-\sum_{j=1}^{m} d_{j}\left(\left|a_{i j}(t)\right| F_{j}+\left|e_{i j}(t)\right| H_{j} \int_{0}^{\tau} k_{i j}(s) d s\right)>0, \quad i=1, \ldots, k, \\
d_{j} B_{j} c_{j}(t)-\sum_{i=1}^{k} \widetilde{d}_{i}\left(\left|\widetilde{a}_{j i}(t)\right| \widetilde{F}_{i}+\left|\widetilde{e}_{j i}(t)\right| \widetilde{H}_{i} \int_{0}^{\sigma} \widetilde{k}_{j i}(s) d s\right)>0, \quad j=1, \ldots, m,
\end{gathered}
$$

then system (5.11) has a unique $\omega$-periodic solution which is globally exponentially stable. 
Remark 5.3. For the $\omega$-periodic BAM neural network model (5.11), R. Wu obtained the existence and global exponential stability of a $\omega$-periodic solution assuming that, for some $\widetilde{d}=\left(\widetilde{d}_{1}, \ldots, \widetilde{d}_{k}\right)>0$ and $d=\left(d_{1}, \ldots, d_{m}\right)>0$,

$$
\begin{gathered}
\widetilde{d}_{i} \widetilde{B}_{i} \widetilde{c}_{i}^{-}-\left(1+\widetilde{B}_{i} \widetilde{c}_{i}^{+} \omega\right) \sum_{j=1}^{m} d_{j}\left(a_{i j}^{+} F_{j}+e_{i j}^{+} H_{j} \int_{0}^{\tau} k_{i j}(s) d s\right)>0, \quad i=1, \ldots, k, \\
d_{j} B_{j} c_{j}^{-}-\left(1+B_{j} c_{j}^{+} \omega\right) \sum_{i=1}^{k} \widetilde{d}_{i}\left(\widetilde{a}_{j i}^{+} \widetilde{F}_{i}+\widetilde{e}_{j i}^{+} \widetilde{H}_{i} \int_{0}^{\sigma} \widetilde{k}_{j i}(s) d s\right)>0, \quad j=1, \ldots, m,
\end{gathered}
$$

where, for a real function $g$, we denote $g^{+}:=\sup _{t \in[0, \omega]}|g(t)|$ and $g^{-}:=\inf _{t \in[0, \omega]}|g(t)|$.

Clearly (5.14)-(5.15) implies (5.12)-(5.13) and they are not equivalent. Hence, the above corollary improves strongly the results in [20] about the global exponential stability of the periodic BAM neural network with finite delays.

\section{Conclusion}

We have presented a criterion for the global exponential stability of a general nonautonomous Hopfield neural network model with delays given here by equations (3.10) and (3.19). We also have presented a criterion for the existence and global exponential stability of a periodic solution for the same models.

These criteria are simple to verify, do not involve the use of Lyapunov functionals, and are directly applicable to most of the nonautonomous Hopfield neural network models with finite delays investigated in recent literature.

Roughly speaking, in this paper the results on the global exponential stability have been obtained by assuming that the instantaneous negative feedback terms dominate the delay effect, so that in spite of the delays, the delay differential equations behaves similarly to an ordinary differential equations.

As illustration, we have applied our general results to a significant number of concrete nonautonomous Hopfiel neural network models, and provided immediate sufficient conditions for their global exponential stability.

In a forthcoming work, we shall exploit the ideas beyond our general method to address the global exponential stability of nonautonomous neural network models, (3.10) and (5.1), with unbounded delays.

\section{Acknowledgements}

This research was supported by Fundação para a Ciência e a Tecnologia through the research center CMAT (J.J. Oliveira). The authors thank the referee for valuable comments.

\section{References}

[1] P. Baldi \& A. F. Atiya, How delays affect neural dynamics and learning, IEEE Trans. Neural Networks 5 (1994) 612-621.

[2] J. Bélair, Stability in a model of a delayed neural network, J. Dynam. Differential Equations 5 (1993) 607-623. 
[3] S. A. Campbell, Delay independent stability for additive neural networks, Differential Equations Dynam. Systems 9 (2001) 115-138.

[4] J. Cao \& L. Wang, Exponential stability and periodic oscillatory solution in BAM networks with delays, IEEE Trans. Neural Networks 13 (2002) 457-463.

[5] M. Cohen \& S. Grossberg, Absolute stability of global pattern formation and parallel memory storage by competitive neural networks, IEEE Trans. Syst. Man Cybern. 13 (1983) 815-826.

[6] T. Faria \& J.J. Oliveira, Local and global stability for Lotka-Volterra systems with distributed delays and instantaneous negative feedbacks. J. Differential Equations 244 (2008) 1049-1079.

[7] T. Faria \& J.J. Oliveira, Boundedness and global exponential stability for delayed differential equations with applications, Appl. Math. Comput. 214 (2009) 487-496.

[8] J. K. Hale \& S. M. Verduyn Lunel, Introduction to Functional Differential Equations, Springer-Verlag, New-York, 1993.

[9] J.J. Hopfield, Neural networks with graded response have collective computational properties like those of two-state neurons, Proc. Natl. Acad. Sci. 81 (1984) 3088-3092.

[10] H. Jiang, J. Cao \& Z. Teng, Dynamics of Cohen-Grossberg neural networks with timevarying delays, Phys. Lett. A 354 (2006) 414-422.

[11] H. Jiang, Z. Li \& Z. Teng, Boundedness and stability for nonautonomous cellular neural networks with delays, Phys. Lett. A 306 (2003) 313-325.

[12] A. N. Kolmogorov \& S. V. Fomin, Introductory Real Analysis, Dover Publications Inc., New-York, 1970.

[13] B. Kosko, Bidirectional associative memories, IEEE Trans. Systems Man Cybern. 18 (1988) 49-60.

[14] C. M. Marcus \& R. M. Westervelt, Stability of analog neural networks with delay, Phys. Rev. A 39 (1989) 347-359.

[15] J. J. Oliveira, Global asymptotic stability for neural network models with distributed delays, Math. Comput. Model. 50 (2009) 81-91.

[16] J. J. Oliveira, Global stability of a Cohen-Grossberg neural network with both timevaring and continuous distributed delays, Nonlinear Anal. RWA 12 (2011) 2861-2870.

[17] M. Tan \& Y. Tan, Global exponential stability of periodic solution of neural network with variable coefficients and time-varying delays, Applied Mathematical Modelling 33 (2009) 373-385.

[18] P. Van Den Driessche, X. Zou, Global stability in delayed Hopfield neural network models, SIAM J. Appl. Math. 58 (1998) 1878-1890.

[19] J. Wu, Introduction to Neural Dynamics and Signal Transmission Delay, Walter de Gruyter, New York, 2001. 
[20] R. Wu, Exponential convergence of BAM neural networks with time-varying coefficients and distributed delays, Nonlinear Anal. RWA 11 (2010) 562-573.

[21] Q. Zhang, X. Wei \& J. Xu, Exponential stability for nonautonomous neural networks with variable delays, Chaos Solitons \& Fractals 39 (2009) 1152-1157.

[22] Q. Zhang, X. Wei \& J. Xu, Delay-dependent exponential stability criteria for nonautonomous cellular neural networks with time-varying delays, Chaos Solitons \& Fractals 36 (2008) 985-990. 